\title{
An Experimental Technique for the Dynamic Characterization of Soft Complex Materials
}

\section{Journal Article}

\section{Author(s):}

Thevamaran, Ramathasan; Daraio, C.

Publication date:

2014-10

Permanent link:

https://doi.org/10.3929/ethz-b-000089975

Rights / license:

In Copyright - Non-Commercial Use Permitted

Originally published in:

Experimental Mechanics 54(8), https://doi.org/10.1007/s11340-014-9896-9 


\title{
An Experimental Technique for the Dynamic Characterization of Soft Complex Materials
}

\author{
R. Thevamaran • C. Daraio
}

Received: 22 November 2013 / Accepted: 17 April 2014 /Published online: 13 June 2014

(C) Society for Experimental Mechanics 2014

\begin{abstract}
We describe an experimental technique to study the dynamic behavior of complex soft materials, based on high-speed microscopic imaging and direct measurements of dynamic forces and deformations. The setup includes high sensitivity dynamic displacement measurements based on geometric moiré interferometry and high-speed imaging for $i n-s i t u$, full-field visualization of the complex micro-scale dynamic deformations. The method allows extracting dynamic stress-strain profiles both from the moiré interferometry and from the high-speed microscopic imaging. We discuss the advantages of using these two complementing components concurrently. We use this technique to study the dynamic response of vertically aligned carbon nanotube foams subjected to impact loadings at variable deformation rates. The same technique can be used to study other micro-structured materials and complex hierarchical structures.
\end{abstract}

Keywords Complex materials $\cdot$ Hierarchical materials . Impact testing $\cdot$ Geometric moiré $\cdot$ High-speed microscopic imaging

\footnotetext{
R. Thevamaran $\cdot$ C. Daraio

Engineering and Applied Science, California Institute of Technology, Pasadena, CA 91125, USA

R. Thevamaran

e-mail: rthevama@caltech.edu

R. Thevamaran · C. Daraio $(\bowtie)$

Department of Mechanical and Process Engineering, Swiss Federal Institute of Technology (ETH Zurich), Zurich 8092, Switzerland

e-mail: daraio@ethz.ch
}

\section{Introduction}

Complex materials have hierarchically organized constituents that are either self-assembled naturally (e.g. natural and biological materials such as wood, bone, teeth and seashells) or engineered to have constituents ranging from nanometers to millimeters in size (e.g. micro and nano structured metamaterials, vertically aligned carbon nanotube (VACNT) structures, etc.) [1]. Biological complex materials are characterized by optimal mechanical properties that can combine strength, stiffness and toughness, and can serve as models for the design of synthetic composites [2,3]. Synthetic complex materials outperform conventional materials by exhibiting exceptional multi-functional properties (for example, superior thermoelectric, piezoelectric and optoacoustic properties) and can achieve effective mechanical properties not found in natural materials (for example, negative elastic modulus, phononic band gaps and high specific energy absorption) [1, 2, 4-6]. Soft complex materials undergo large finite deformations with shape changes when subjected to an external stress field and may exhibit distinct deformation characteristics in different length and time scales [7]. Understanding the behavior of complex soft materials in a broad range of strain-rates from $10^{-8}$ to $10^{8} \mathrm{~s}^{-1}$ is of great interest to material scientists and engineers to: (i) understand the fundamental mechanisms that govern the mechanics and the resultant material behavior; (ii) synthesize novel materials with physical properties that can be tailored for specific applications.

At very low rates $\left(10^{-8}-10^{-6} \mathrm{~s}^{-1}\right)$, creep and stress relaxation experiments subjected to prolonged loading conditions have been used to study the viscoelastic and long-term behavior $[8,9]$. At quasi-static rates $\left(10^{-4}-10^{\circ} \mathrm{s}^{-1}\right)$ tensile, compression, indentation and shear experiments have been adapted to understand complex deformation responses of hierarchical structured materials [10]. At higher rates, 
dynamic mechanical analysis (DMA) is a commonly used technique to obtain the material parameters such as storage and loss modulus [11]. Nonetheless, DMA has been limited to small strain amplitudes and has not been successful in characterizing inhomogeneous materials with intrinsic gradient in functional properties. At yet higher rates, drop ball impact testing [12], projectile impact experiments [13] and shock wave excitation [4] have been used to study the rate dependent materials behavior. For each experimental technique used, appropriate force and displacement measurement systems have been designed and improved. The most common experimental methods and detection systems used to test materials at different rates of deformations [14-16] are summarized in Fig. 1.

Non-contact, optical visualization methods have been the most widely adopted and rapidly evolving techniques for insitu imaging and quantitative displacement measurements. Recent advancements in high-speed imaging and digital image correlation methods [17-19] enabled the mechanical, damage and fracture characterization of macroscopic complex materials, at various rates of deformations [20-22]. At smaller scales, quasi-static nano-indentation experiments performed inside a scanning electron microscope (SEM) have been used to visualize and characterize the localized microscale deformation [23]. Optical interferometry techniques, such as moiré interferometry, speckle interferometry, holographic interferometry and shearing interferometry, have been widely used for non-contact dynamic displacement measurements [14].

Despite all the advances in experimental testing and measurement techniques, the characterization of the dynamic behavior of soft complex materials remains challenging. This is due to their hierarchical microstructure, which requires the characterization of deformation phenomena occurring at both micro- and macro-scales. In addition, the bulk material's behavior of these samples is affected by the presence of large three-dimensional deformations and local effects arising from defects and intrinsic functional property gradients. In this

\begin{tabular}{c|c|c} 
Experimental method & $\begin{array}{c}\text { Strain-rate } \\
\left(\mathbf{s}^{-1}\right) \\
10^{-8}\end{array}$ & $\begin{array}{c}\text { Displacement sensing: } \\
\text { example optical techniques }\end{array}$ \\
\hline $\begin{array}{c}\text { Creep, Relaxation } \\
\text { experiments }\end{array}$ & $10^{-4}$ & Time-lapse photography \\
\hline $\begin{array}{c}\text { Quasistatic Compression, } \\
\text { Tension, Shear experiments, } \\
\text { Nano-indentation }\end{array}$ & $10^{0}$ & $\begin{array}{c}\text { Videography, In-situ scanning } \\
\text { electron microscopy, Digital image } \\
\text { correlation, Interferometry } \\
\text { techniques }\end{array}$ \\
\hline $\begin{array}{c}\text { Dynamic mechanical analysis, } \\
\text { Specialized instrumentations }\end{array}$ & $10^{2}$ & $\begin{array}{c}\text { High-speed imaging, } \\
\text { Interferometry techniques }\end{array}$ \\
\hline $\begin{array}{c}\text { Drop-weight impact } \\
\text { Hopkinson pressure bar }\end{array}$ & $10^{4}$ & $\begin{array}{c}\text { High-speed imaging, } \\
\text { Interferometry techniques }\end{array}$ \\
\hline Plate impact & $\begin{array}{c}\text { Ultra-high-speed imaging, } \\
\text { Digital image correlation } \\
\text { Interferometry techniques }\end{array}$ \\
\hline experiments & $10^{8}$ & . \\
\hline
\end{tabular}

Fig. 1 Overview of mechanical testing techniques and of the relevant optical measurements manuscript, we present an experimental technique to characterize the dynamic response of complex soft materials. The technique measures the global dynamic constitutive response, using dynamic force sensors and non-contact optical interferometry (moiré). This allows for the characterization of the samples' large deformations. The local dynamic effects, occurring at the micro-scale, are captured by the in-situ visualization using high-speed microscopic imaging. The ability to acquire microscopic images also facilitates the characterization of thin samples. We target dynamic characterization at nominal strain rates ranging between $10^{2}$ and $10^{4} \mathrm{~s}^{-1}$.

In the following sections, we provide a detailed account on the experimental set up (section 2), VACNT specimen fabrication (section 3), data reduction methodologies (section 4), comparison of the dynamic behavior of VACNT foams obtained independently from the two displacement measurement components of the experimental setup: geometric moiré interferometry and high-speed microscopic imaging (section 5) and a discussion on the observed dynamic behavior of VACNT foams (section 6).

\section{Experimental Set Up}

The dynamic testing apparatus (Fig. 2(a)) described here has four main components: (i) a striker impact system, (ii) a dynamic force sensor, (iii) a dynamic displacement transducer, and (iv) a high-speed camera with a microscopic lens for visual observation and characterization of the deformation. The details of each of these components are described in the following subsections.

\section{Striker Impact System}

The striker impact system consists of a flat-plunge striker, a frictionless striker guide and a pneumatic cylinder to launch the striker on the guide. We use a flat plunge striker made of Delrin ${ }^{\circledR}$ acetal homopolymer as the loading apparatus. The striker material was chosen to have adequate stiffness to accommodate no or minimal deformation compared to the material being tested. The Delrin ${ }^{\circledR}$ acetal homopolymer has two orders of magnitude higher modulus than the modulus of VACNT foams. Its material properties are: density, $1.41 \mathrm{gcm}^{-3}$, tensile and compressive modulus, $3.10 \mathrm{GPa}$, compressive strength, $110 \mathrm{MPa}$, and Rockwell hardness, $\mathrm{R} 122$ [24]. The main body of the striker is $28.5 \mathrm{~mm}$ long and has a square cross-section (side length $=12.70 \mathrm{~mm}$ ). It has a cylindrical plunge (length $=$ diameter $=6.35 \mathrm{~mm}$ ) with a flat face as the loading front. The flat loading front was chosen to provide uniaxial planar impacts to the specimen. It should be noted that albeit indentation is being used as an indirect method to obtain material properties $[25,26]$, the interaction of sharp indenter tips with structured materials with complex 


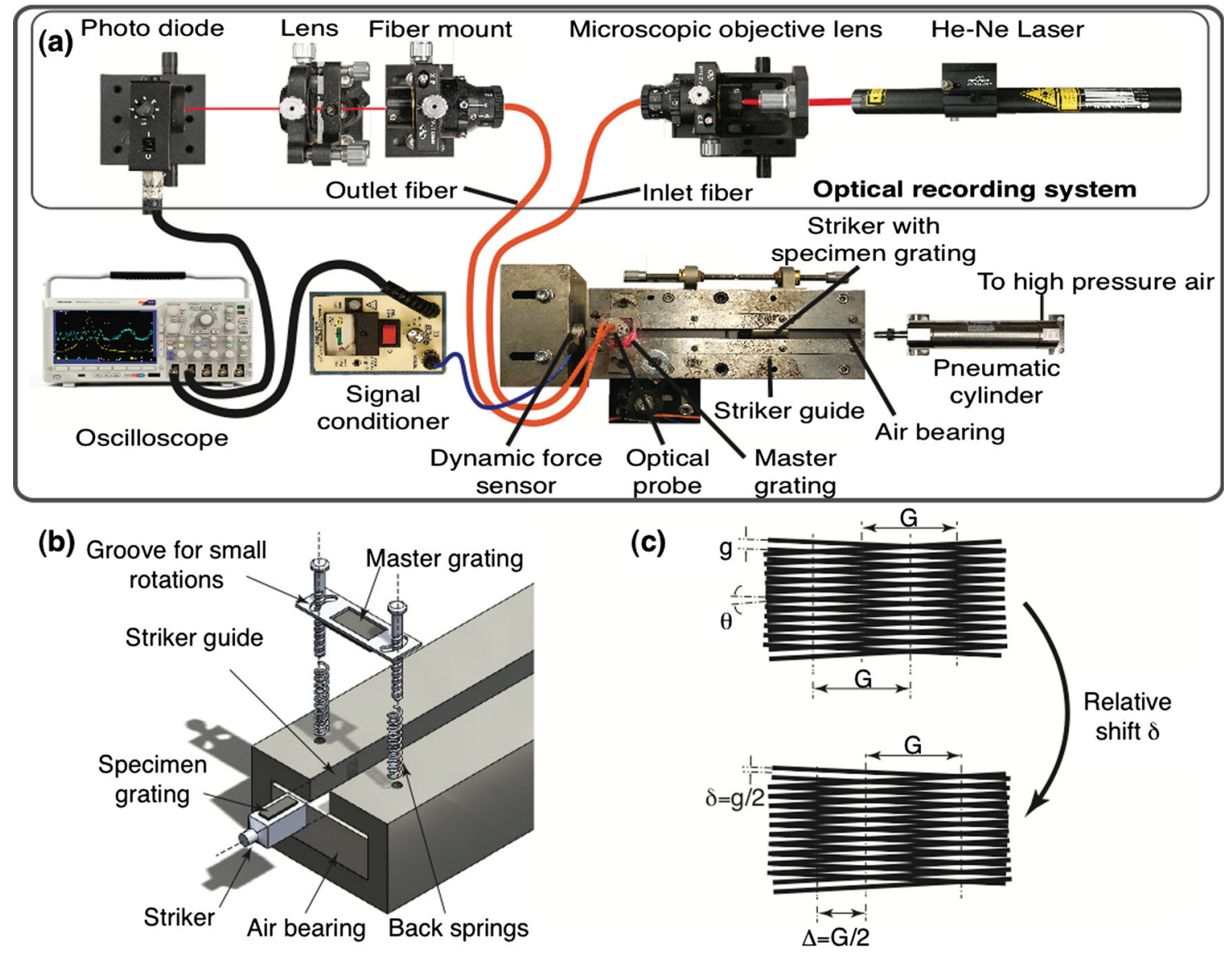

Fig. 2 Geometric moiré interferometry based dynamic testing setup: a experimental set up b schematic of geometric moiré implementation on striker guide assembly $\mathbf{c}$ schematic of geometric moiré fringe formation and fringe shift

microstructure is not well understood [27, 28]. However, if a dynamic indentation experiment is desired, the loading front of the striker in our system can be modified to accommodate spherical indenters or sharp indenter shapes, like Vickers and Berkovich [29]. The square cross-section of the striker body was selected to prevent any rotation that can significantly impair the quality of the moiré fringes.

A stainless steel double-acting pneumatic cylinder (McMaster-Carr, with bore, $26.98 \mathrm{~mm}$ and stroke, $38.10 \mathrm{~mm}$ ) was used to launch the striker on a frictionless striker guide. An air regulator coupled with a pressure gauge varied the pressure in the pneumatic cylinder from 10 to $60 \mathrm{psi}$ (68.9-414 kPa) to control the striker launching speed from 1 to $6 \mathrm{~ms}^{-1}$. The striker guide was designed to propel the striker straight to $200 \mathrm{~mm}$ travel distance. A spacing of $\sim 10 \mu \mathrm{m}$ was left between the striker and the confining guide walls to reduce friction. In addition, a $\sim 5 \mu \mathrm{m}$ thin layer of air was introduced between the striker and the base of the guide using flat rectangular air-bearings to facilitate frictionless traveling of striker. The air bearing $\left(N e w w a y{ }^{\circledR}\right)$ is made of a micro-porous carbon media and supplied with high-pressure air to form the thin layer of air. This design of the frictionless striker guide dramatically enhanced the repeatability of the striker's impact velocities compared to conventional low-friction material assemblies.

\section{Force Sensor}

We use PCB Piezotronics Quartz ICP® impact force sensors (model 200B02 and 200B03) for measuring the transient signals during the impacts. Quartz transducers are desired for dynamic applications due to the high natural resonant frequency of quarts $(>1 \mathrm{MHz})$. Similar transducers have been used in several other dynamic testing systems [25, 30, 31]. The discharge time constants $(\tau)$ of the sensors used in our experiments are $\tau>500 \mathrm{~s}$ for the 200B02 [32] and $\tau>2,000 \mathrm{~s}$ [33] for the 200B03, which are well above the loadingunloading time of our experiments $(\sim 1 \mathrm{~ms})$. These sensors have positive polarity for compression, they have sensitivities of $11,241 \mathrm{mV} / \mathrm{kN}$ (200B02) and $2248.2 \mathrm{mV} / \mathrm{kN}$ (200B03) and can operate up to $444.8 \mathrm{~N}$ (200B02) and 2,224 N (200B03) with high linearity $[32,33]$. The $I C P ®$ force sensors incorporate a built-in amplifier to convert the high impedance charge output to low impedance signal that is not affected by triboelectric cable noise or contaminants before recording. We conditioned the voltage signal from the dynamic force sensors 
and amplify it (when required) using an $I C P ®$ sensor signal conditioner (PCB Piezotronics model: 480E09; gain x1, x10, $\mathrm{x} 100)$ connected to the force sensor by a low-noise coaxial cable (PCB Piezotronics model: 003C05). The Tektronix DPO3034 oscilloscope records the output signal from the signal conditioner.

The force sensor is mounted firmly on a rigid stainless steel block with heavy mass according to the specifications provided by the PCB Piezotronics. We attach the specimen to the striker and let it directly impact the force sensor. For the impact velocity ranges considered (from 1 to $6 \mathrm{~ms}^{-1}$ ), the force-response time during impact is much larger than the time taken to equilibrate stresses. This implies that the dynamic force measured by the force sensor represents equilibrated stresses. Equilibration of axial stress is essential to ensure that the measured stress represents the average stress in the specimen. Davies and Hunter [34] estimated that the stress equilibration requires three reverberations of the loading wave in the specimen. We estimate this to amount to $230 \mu \mathrm{s}$ in our experiments. This is a conservative estimate using the quasistatically measured modulus of $\sim 10 \mathrm{MPa}$ for a representative VACNT foam with $\sim 0.25 \mathrm{gcm}^{-3}$ density. The longitudinal wave velocity estimated there by is $\sim 200 \mathrm{~ms}^{-1}$ and it will take $\sim 10 \mu$ s for one reverberation in a sample with thickness $\sim 1 \mathrm{~mm}$. Song and Chen [35] showed that for a soft material, the specimen thickness has a profound effect on establishing stress equilibrium-higher the thickness, longer the initial non-equilibrium stress duration is. In addition, they showed that the high loading rate too has an effect on stress equilibrium, but it is less pronounced compared to the thickness of the specimen. The stress equilibration is a critical design consideration for very high loading-rate experiments, like the Kolsky split-Hopkinson bar tests, particularly with higher specimen thicknesses and has low significance in relatively long duration $(\sim 1 \mathrm{~ms})$ loading-unloading experiments with moderate loading-rates and thinner specimens $(\sim 1 \mathrm{~mm})$.

\section{Dynamic Displacement Transducer}

Accurate dynamic displacement measurement in soft, hierarchical and micro-structured samples is a challenging task that requires the development of an ad-hoc measurement system. The complex microstructure and the small characteristic size of the samples make conventional optical techniques unsuitable, due to the current resolution limits of high-speed cameras and the need for powerful illumination sources. The setup we developed includes a dynamic displacement measurement system capable of capturing micro-scale deformation during impact loadings. The measurement system uses high sensitivity geometric moiré fringes to amplify the microscopic dynamic displacements. Among all the different types of moiré techniques, geometric moiré is one of the simplest to implement. The technique relies on the superposition of two identical line-gratings with a small relative angle of rotation to create a geometric amplification of displacement that can be used for high sensitivity measurements [36-39]. Geometric moiré interferometry was also used in dynamic indentation experiments to study the rate-sensitivity of oxygen-free highconductivity (OFHC) copper [25]. Geometric moiré fringes are produced by the interference of two identical line gratings (master and specimen gratings) superimposed with a very small relative angle of rotation [36] (see Fig. 2(b) and (c)). In most applications of geometric moire', the master grating is attached to the experimental fixture and the specimen grating is attached to the sample [36]. The moire fringes produced this way have a pitch, $\mathrm{G}$, that is related to the pitch of the gratings, g, through a purely geometric relationship [36]:

$G=\frac{g}{\theta}$

where $\theta$ is the small relative angle of rotation. The pitch of the grating is the center-to-center spacing between two neighboring dark lines (or white lines), and the half-pitch is the centerto-center spacing between two adjacent dark and white lines. When one grating is displaced by an amount $\delta$ relative to its pair, the resulting moiré fringes move to a new position by $\Delta$ given by [36]:

$\Delta=\delta\left(\frac{G}{g}\right)$

where $\mathrm{G} / \mathrm{g}$ is the amplification factor. Hence, this technique can be interpreted as a displacement amplifier that can be used to sense micro-scale displacements. For example, a grating pair at a $2^{\circ}$ relative angle of rotation amplifies the displacement nearly by a factor of 30 .

We implement this technique as follows: the master grating is mounted stationary on the striker guide and the specimen grating is rigidly attached to the striker using a 5 min epoxy. The specimen grating was attached on the striker, and not on the samples directly, because of the samples' small size and their complex, large deformations under impact. The rigidbody translation of the striker (and attached specimen grating) relative to the stationary master grating produces the shift in the geometric moiré fringes. When the striker is in contact with the sample, the shift in the moire' fringes measures the dynamic deformation of the specimen. The master grating was mounted on the striker guide with back-loaded spring supports as shown on the Fig. 2(b). This arrangement provides the flexibility to tune the moiré fringe amplification factor by varying the relative angle between the specimen and master gratings.

The gratings used have an identical rounchi ruling frequency of 40 cycles $/ \mathrm{mm}$ resulting in a pitch $g=25 \mu \mathrm{m}$. The nominal sensitivity of the geometric moiré technique is equal 
to the pitch of its grating (corresponds to peak to peak distance on the intensity modulation of the moiré fringes). However, we were able to measure displacements at $6.25 \mu \mathrm{m}$ (quarterpitch) resolution through data reduction as explained in Sec. 3. Both the specimen grating (reflective) and the master grating (transparent) were produced by Applied Images Inc. The reflective specimen grating was created by first coating a glass substrate with a specular-reflective thin layer of aluminum and then printing precise blue-chrome ronchi rulings using a semiconductor pattern generator device. Similarly, the transparent grating was produced by patterning blue-chrome lines on a transparent glass substrate. During measurement, the focused laser light from the optical probe passes through the transparent master grating, reflects on the reflective specimen grating and passes again through the transparent grating before being recollected back into the optical probe. The resulting multiplicative intensity, $I$, of the emergent light becomes [36]:

$I=I_{0}(T(x, y))^{2} R(x, y)$

where $R$ is the reflectance at each $(x, y)$ point of the reflective grating and $T$ is the transmission at each $(x, y)$ point of the transparent grating. Even though the best multiplicative moiré pattern is obtained when the two gratings are in contact, no deterioration of the pattern is noticeable when the gap between the two gratings is much smaller than $\mathrm{g}^{2} / \lambda$ where $\lambda$ is the wavelength of light and $g$ is the pitch of the gratings [36]. If a red-laser light source $(\lambda=632.8 \mathrm{~nm})$ is used on a grating pair with $25 \mu \mathrm{m}$ pitch, the gap between the specimen and master gratings should be smaller than $1 \mathrm{~mm}$ to produce moiré fringes of sufficient quality for measurements. A relatively large gap is essential during impact experiments, to ensure no damage to the gratings. This becomes a limiting factor in increasing the grating frequency, and the related displacement resolution.

The optical system (Fig. 2) that is used to produce coherent light and record the intensity modulation of the geometric moiré fringe shift has several optical components (the essential design parameters are shown within parentheses): A coherent light source (He-Ne Laser; with a wavelength of $632.8 \mathrm{~nm}$; power $21 \mathrm{~mW}$ ); an objective lens to focus the laser light into the inlet fiber; an inlet fiber (with a $50 \mu \mathrm{m}$ core diameter) that directs the light to the optical probe; an optical probe with two internal miniature lenses $(5 \mathrm{~mm}$ field depth; $30 \mathrm{~mm}$ probe to specimen distance; and a focused spot size on the specimen with a $600 \mu \mathrm{m}$ diameter; numerical aperture 0.15 ) to focus the light onto the grating and recollect the emergent light from the grating; outlet fiber (core diameter of $300 \mu \mathrm{m}$ ) that transports the collected emergent light to photo detector; an achromatic lens to focus the light from the outlet fiber onto the photo detector; $\mathrm{Si}$ switchable gain photo detector (wavelengths between 350 and 1,100 nm; $17 \mathrm{MHz}$ bandwidth; detection area of $13 \mathrm{~mm}^{2}$ ) to detect the light intensity from the optical probe and convert into voltage signal; and an oscilloscope (Tektronix DPO3034; analog bandwidth, $300 \mathrm{MHz}$; sample rate, $2.5 \mathrm{GS} / \mathrm{s}$; record length, 5 million points) to record the signal received by the photo detector. In addition, several optomechanics components such as multimode fiber couplers, linear stages, lens mounts, laser mount, optical posts, post holders and mounting bases are used in the setup. A photo detector with fine rise-time was selected in order to record the light intensity modulation. For an impact velocity of $5 \mathrm{~ms}^{-1}$ and a grating pitch g, $25 \mu \mathrm{m}$, the time for the dark-bright intensity transition (corresponding to a half-pitch movement) is $2.5 \mu \mathrm{s}$. We selected a photo detector with rise time $\sim 60 \mathrm{~ns}$ to have enough frequency response to record the moiré fringe shifts. It should be noted that a noncoherent, high intensity, white light source with narrow beam size can be used in place of coherent laser light source for grating frequencies up to 40 cycles $/ \mathrm{mm}$. In white light illumination conditions, when the grating frequency increases beyond 40 cycles $/ \mathrm{mm}$, the contrasts of the recorded intensity modulations decrease below the noise level and the sensitivity of the moire' setup is not sufficient for data acquisition. The use of a coherent light source improves the fringe contrast and can be used to achieve higher measurement resolution [14].

\section{High-speed Microscopic Imaging}

We use a Vision Research's Phantom V1610 high-speed camera coupled to a microscopic lens (Infinity long-distance microscope system model $K 2 / S C$ ) to observe and characterize dynamic deformations. The camera has a CMOS (35.8 $\mathrm{mm} \times$ $22.4 \mathrm{~mm}$ ) sensor with $1,280 \times 800$ pixels maximum spatial resolution and operates up to 1 million frames-per-second (fps) frame-rate (with a temporal resolution of $1 \mu \mathrm{s}$ ). A Karl Storz Xenon Nova 300 high-intensity cold light lamp (which includes a 300 Watt Xenon lamp) connected to a Karl Storz liquid light guide cable was used to illuminate the specimen. The light sensitivity of the camera to low-intensity reflected light from our black samples proved to be a limitation in reaching high temporal resolutions (i.e., high frame-rates). We operate the camera at a frame rate of $\sim 150,000 \mathrm{fps}$, at a resolution of $128 \times 256$ pixels, focused on a physical window of $\sim 1.8 \mathrm{~mm} \times 3.5 \mathrm{~mm}$. The Phantom Cine View 2.14 software is used to control the camera. The camera is synchronized with the rest of the experimental set up and its acquisition is triggered by the trigger-signal from the oscilloscope. The Image systems's TEMA motion analysis software was used to analyze the image sequence. This software is capable of analyzing the image sequence using a digital image point correlation algorithm with an accuracy of 1 pixel. The high-speed image sequence provides information to understand the complex dynamic deformation of the VACNT foams that cannot be observed with the geometric moiré interferometry alone. 


\section{VACNT Foam Specimen Fabrication}

We study the dynamic response of VACNT foams using the described testing system. To synthesize the CNT foams we use a floating catalyst thermal chemical vapor deposition (tCVD) process [40]. The carbon source (toluene), and catalyst pre-cursor (ferrocene) mixed at $0.2 \mathrm{~g} \mathrm{ml}^{-1}$ were injected at $1 \mathrm{ml} \mathrm{min}{ }^{-1}$ into the carrier gas (argon) flowing at $800 \mathrm{sccm}$. Oxidized silicon substrates, placed inside a $15 \mathrm{~cm}$ heating zone of a quartz furnace tube, were used as growth substrates. The furnace tube was maintained at $827^{\circ} \mathrm{C}$ and atmospheric pressure throughout the synthesis. VACNT foams synthesized using this tCVD process have been shown to present a hierarchical fibrous morphology (40 and Fig. 3) in which bundles of multi-walled carbon nanotubes (MWCNT) are vertically aligned at the meso-scale and form a forest-like system in the micro- and nano-scales. The physical properties of the samples synthesized for this work are provided in Table 1.

In addition, these VACNT foams are characterized by an intrinsic gradient in functional properties, such as density and stiffness, which leads to sequential buckling when the samples are subjected to compressive loadings [23, 40, 41]. The typical curves obtained by testing the VACNT foams are shown in Fig. 4.

\section{Data Reduction Methodology and Analysis}

During impact, the force-time history (Fig. 4(a)) and the moiré fringe intensity modulation history (Fig. 4(b)) are recorded using the oscilloscope. The recorded data is smoothed for noise using a low-pass filter before analysis. A striker displacement of $25 \mu \mathrm{m}$ (equivalent to the relative movement of a pitch of the gratings) corresponds to a full sinusoidal cycle in the intensity

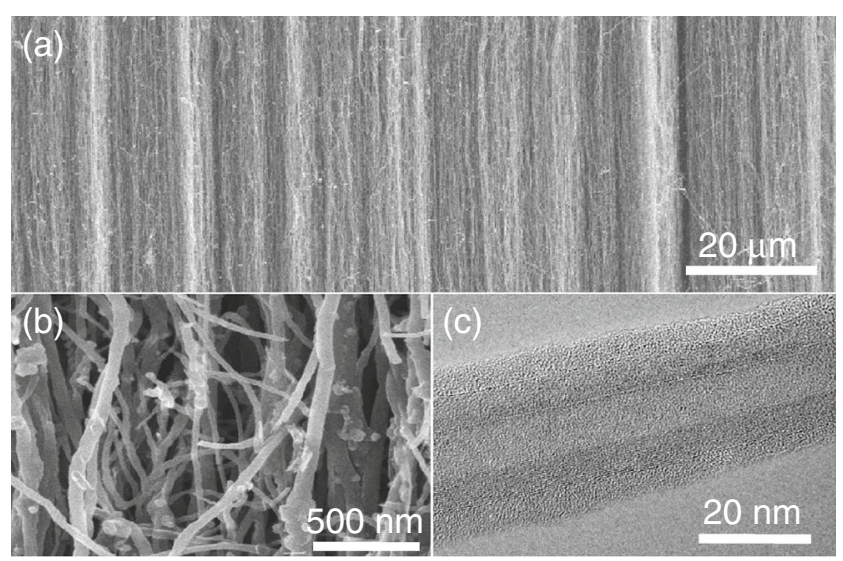

Fig. 3 Hierarchical morphology of VACNT foams: a SEM image of the vertically aligned bundles of CNTs, scale bar $20 \mu \mathrm{m}$ b SEM image of the forest like system in micro and nano scales, scale bar $500 \mathrm{~nm}$ c TEM image of an individual multi-walled carbon nanotube (MWCNT), scale bar $20 \mathrm{~nm}$
Table 1 Physical (white background) and mechanical (gray background) properties of the VACNT foams tested

\begin{tabular}{|l|c|c|}
\hline \multicolumn{1}{|c|}{ Property/parameter } & Specimen $(\mathrm{A})$ & Specimen \\
\hline Diameter $(\mathrm{mm})$ & 5 & 5 \\
\hline Height $(\mathrm{mm})$ & 1.013 & 1.106 \\
\hline Bulkdensity $\left(\mathrm{gcm}^{-3}\right)$ & 0.258 & 0.232 \\
\hline Impact velocity $\left(\mathrm{ms}^{-1}\right)$ & 2.57 & 4.44 \\
\hline Rebound velocity $\left(\mathrm{ms}^{-1}\right)$ & 1.42 & 2.41 \\
\hline Nominal strain rate $\left(\mathrm{s}^{-1}\right)$ & 2537 & 4014 \\
\hline Coefficient of restitution & 0.55 & 0.54 \\
\hline Peakstress $(\mathrm{MPa})$ & 7.59 & 17.28 \\
\hline Maximum strain & 0.41 & 0.55 \\
\hline Energy dissipation $\left(\mathrm{MJm}-{ }^{-}\right)$ & 0.94 & 3.20 \\
\hline Loading modulus $(\mathrm{MPa})$ & 9.93 & 10.96 \\
\hline Unloading modulus $(\mathrm{MPa})$ & 204.93 & 331.14 \\
\hline
\end{tabular}

modulation recording (Fig. 4(b)). Hence, a peak to peak (or valley to valley) distance on the time axis (Fig. 4(b)) corresponds to the time taken for the movement of a pitch $(25 \mu \mathrm{m})$. Similarly, peak to valley (or valley to peak) distance corresponds to a half-pitch $(12.5 \mu \mathrm{m})$. Differentiating the intensity data with respect to time and finding the peak and/or valley locations of that intensity-gradient profile allow us to find the time taken for a quarter-pitch movement $(6.25 \mu \mathrm{m})$. As a result, a simple fringe counting produces the displacement history data (Fig. 4(c)) with a resolution of $6.25 \mu \mathrm{m}$.

Errors associated with data analysis are estimated to be much lower than a quarter-pitch. An additional advantage of using geometric moiré as a displacement transducer is the availability of the velocity-time history (Fig. 4(c)). Impact, rebound velocities, displacement-rate, and nominal strain-rate during deformation are all readily available from the data. We use a central differentiation scheme to derive the velocity-time history from the displacement-time history.

To obtain the complete force-displacement profiles, the initial and final points of loading have to be found. We assume the noise level of the data recorded from the dynamic force sensor to be less than 100th of the force amplitude. We cut the tails of the force-time profile below this level at both ends and find the initial loading point from the initial gradient of the force-displacement data. After unloading, we measure the final (recovered) displacement from the high-speed image sequence. The input from the high-speed camera images is necessary in this case, since the tested samples rapidly unload below 100th of the force amplitude, and continue to recover thereafter until detaching from the force-sensor.

The dynamic stress-strain diagram (Fig. 4(d)) can be obtained from the force-displacement profile normalizing the force by the area of the specimen (nominal axial stress) and the displacement by the initial height of the specimen (nominal or engineering strain). Dynamic material properties such as the loading and unloading moduli, peak stress and maximum strain can be obtained from the dynamic constitutive response. From the maximum and recovery strains, the percentage recovery of foam-like energy absorbing materials can be calculated. Percentage recovery provides a measure for the resilience of the materials. The area included by the hysteresis 
Fig. 4 Experimental measurements of an impacted VACNT foam and data reduction: a force history $\mathbf{b}$ intensity modulation of moiré fringes c displacement and velocity histories d dynamic constitutive response
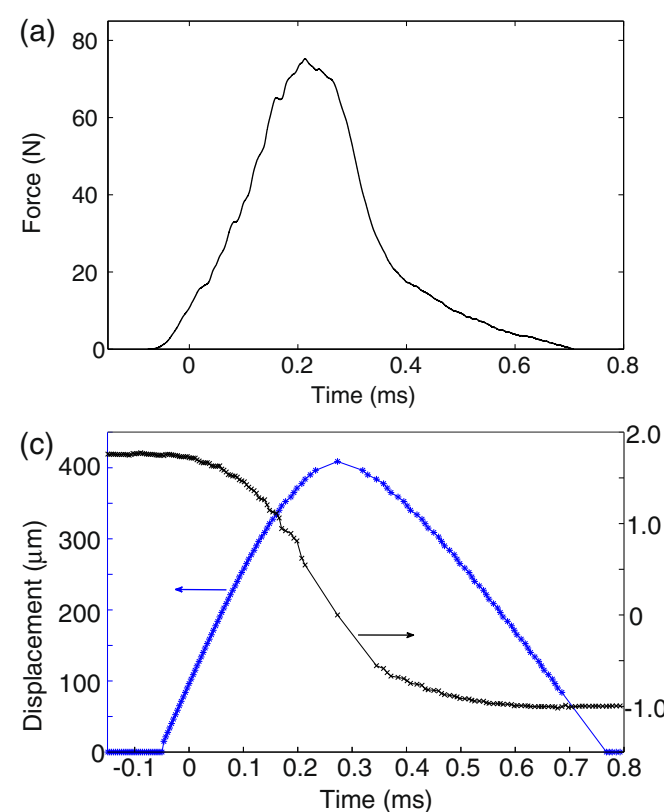
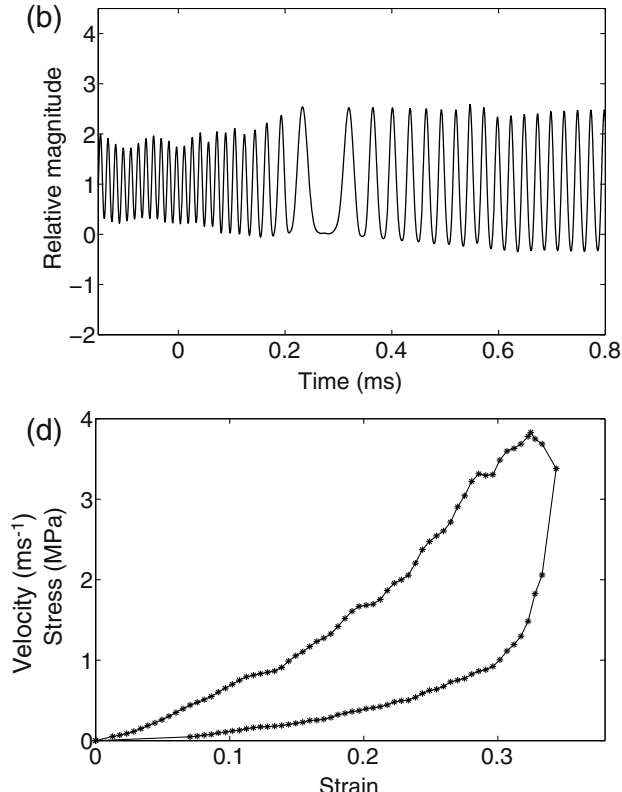

of the stress-strain diagram provides the energy dissipated by the material. From the ratio of rebound to impact velocities, the restitution coefficient which represents the elasticity of the impact can be obtained. These dynamic mechanical properties obtained for the two VACNT foams tested are summarized in Table 1. The dynamic stress-strain profiles corresponding to various loading-rates can be used to study the rate-sensitivity of the test material. Hence this experimental setup allows to quantitatively studying impact response of energy absorbing materials as well as the fundamental micro-scale deformation characteristics of complex soft materials.

\section{Comparison of Displacement Acquisition Methods}

In this section, we compare the dynamic constitutive response obtained from the two independent displacement measurements: (i) geometric moiré interferometry and (ii) high-speed microscopic imaging. Figure 5 shows the comparison of the dynamic constitutive response of two different VACNT foams impacted at $2.57 \mathrm{~ms}^{-1}$ (Strain rate: 2,537 $\mathrm{s}^{-1}$ ), Specimen (A) (Fig. 5(a)), and $4.44 \mathrm{~ms}^{-1}$ (Strain rate: $4,014 \mathrm{~s}^{-1}$ ), Specimen (B) (Fig. 5(b)). Here the nominal strain rates are calculated by dividing the impact velocity by the initial specimen height.

The displacement resolution of the geometric moire interferometer is fixed by the grating pitch selected (and it is equal to $6.25 \mu \mathrm{m})$. On the contrary, the displacement resolution obtained processing the high-speed image sequence depends primarily on three parameters: the spatial resolution of the images, the temporal resolution (frame-rate) and the rate of displacement (or the velocity) of the striker. The displacement resolution of our high-speed camera varied between 10 and $40 \mu \mathrm{m}$, for striker velocities between 1 and $6 \mathrm{~ms}^{-1}$. In all cases tested, the moire interferometry method reached a better displacement resolution and was able to capture stress fluctuations more accurately than the high-speed camera (Fig. 5). The geometric moiré interferometry measured a larger maximum strain than the high-speed imaging technique, which can be attributed to tilting of the striker at the end of loading phase. These deviations were calculated to be less than $5 \%$ of the total deformation.

The main advantage of using microscopic, high-speed image acquisition in the setup is the full field visualization of the samples' deformation. Complex materials often present strain localization, instabilities and sequential deformations phenomena that would not be easily recognized or captured by direct global strain measurements. High-speed imaging enables the in-situ identification and characterization of the deformation mechanisms. For example, the series of frames extracted from the high-speed microscopic image sequence (Fig. 5(c)) demonstrate the ability to identify the formation of localized sequential buckling during loading and the recovery of the VACNT sample. The high-speed image sequence on Fig. 5(c) corresponds to the VACNT foam (specimen (B)) impacted at $4.44 \mathrm{~m} / \mathrm{s}$ and imaged at $128 \times 256$ pixel resolution and 150,000 fps frame-rate.

\section{Material Response}

When the sample is first impacted with the striker, the compressive stress rises linearly with the strain. This deformation regime is followed by a nonlinear region primarily governed by the progressive buckling. The VACNT foams are 


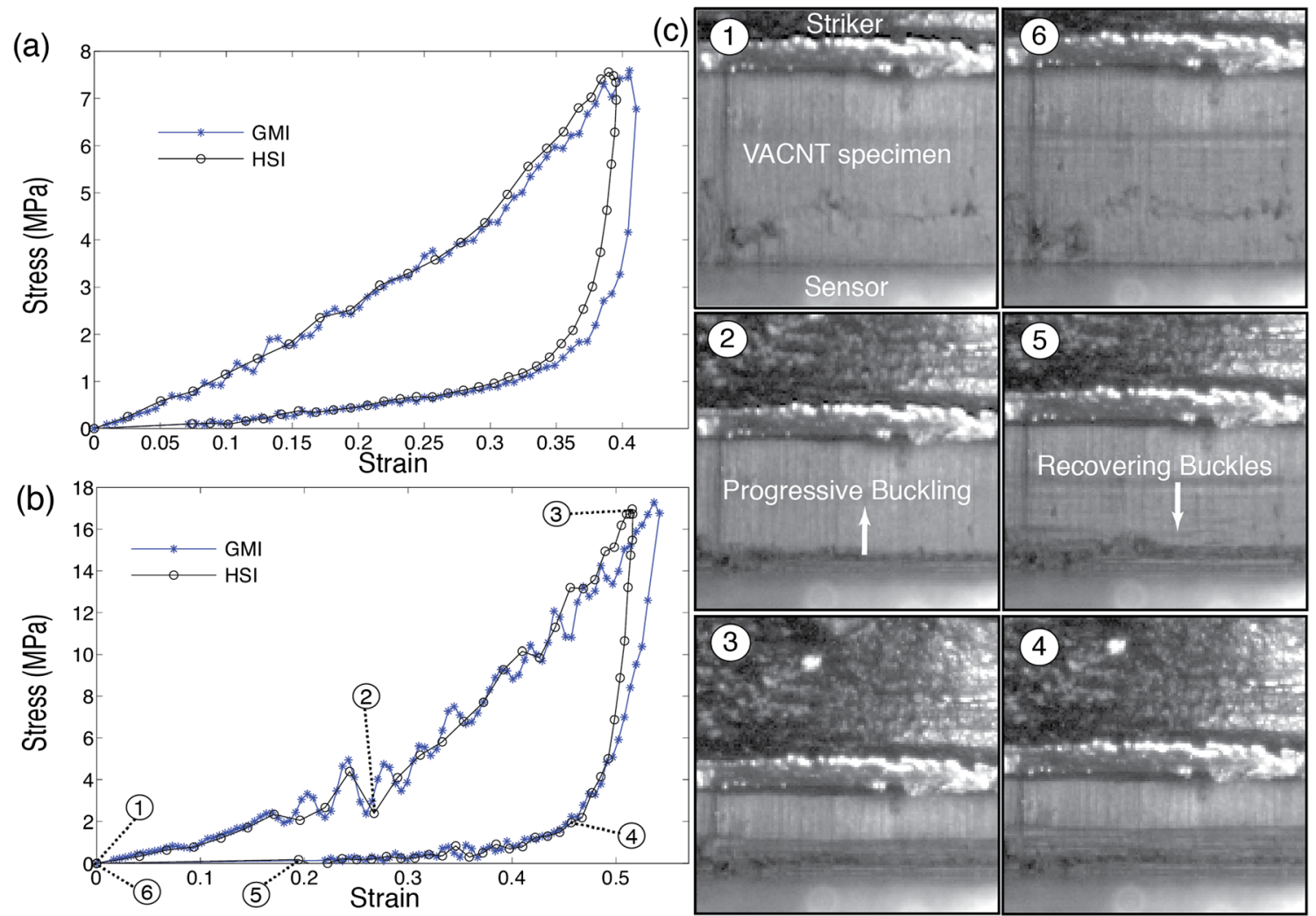

Fig. 5 Comparison of dynamic constitutive responses obtained with geometric moire interferometry (GMI) and high-speed imaging (HSI) at impact velocities a $2.57 \mathrm{~ms}^{-1} \mathbf{b} 4.44 \mathrm{~ms}^{-1} \mathbf{c}$ deformation micrographs of specimen (B) obtained with the high-speed camera

characterized by an intrinsic density gradient along their height that is induced by the tCVD synthesis process (see section 3). This graded functional properties and the fibrous morphology of the foam leads to the formation of collective buckles of the VACNT bundles, which nucleate at the soft side of the specimen at the end of the initial linear stress-strain region. This localization of the deformation occurred independently of the sample's loading side, suggesting that the phenomenon derives from the intrinsic material property gradient.

As the specimen is compressed further, the stress rises with frequent instabilities until the striker reaches a zero velocity. The instabilities characterized by the negative slopes (or stress drops) in the stress-strain or stress-time curves correspond to the formation of buckles (see e.g., Fig. 5(c) image 2-3). This correlation can be seen in our experiments, however, limits arise when the size of the instabilities is below the spatial resolution of our imaging system and/or when the events progress too rapidly to be captured by the camera. A similar one-to-one correlation of instabilities in the force-history profiles with the formation of buckles has been reported for quasistatic compression tests [23]. It should be noted that due to these progressive instabilities, the peak stress reached during impact does not necessarily correspond to the maximum strain. In addition, the material exhibits a stiffening response, in which the compressive stress nonlinearly increases with the strain, as the specimen is compressed. This stiffening response arises from the increasing density gradient along the height of the specimen.

Once the maximum compressive strain is reached, as the striker reaches a zero velocity (Fig. 5(c) image 3), the VACNT foam starts releasing the stored energy and begin pushing the striker back. During this unloading phase, the unloading stress-strain path differs from the loading path, exhibiting a hysteresis. This deviation is evident particularly in the first $10 \%$ of the unloading strain, which corresponds to the progressive recovery of the buckles. When the specimen detaches from the force sensor along with the striker, a full recovery of the specimen is observed (Fig. 5(c) image 6). This demonstrates the high resilience of the VACNT foams to impact.

The hysteresis included within the loading-unloading stress-strain loop accounts for the energy dissipated during the impact. The fundamental energy dissipation mechanisms and the reasons behind the presence of the hysteresis are still not well understood. Earlier studies suggested that the hysteresis derives from the presence of CNT-buckling [41], changes in the orientation or waviness of the individual nanotubes during the loading-unloading cycle [42] and friction between the individual and bundles of the tubes [41]. Using quasistatic compression cycles it was found previously that the energy dissipated by the VACNT foams deforming up to $80 \%$ strain is more than 200 times higher than the energy dissipated by the commercial foams with similar densities [43]. The global 
dynamic behavior in the impact velocity range considered $\left(1-6 \mathrm{~ms}^{-1}\right)$ is similar to the response observed in quasistatic compression cycles [23, 40, 41].

\section{Conclusions}

In summary, we have developed an experimental technique to study the dynamic behavior of soft, complex materials subjected to impact loading. We use this technique to measure the dynamic response of VACNT foams as example. The VACNT foam specimens tested exhibited a nonlinear dynamic stressstrain behavior with a hysteretic dissipation. The in-situ visualization using the high-speed microscopic camera revealed progressive collective buckling of the VACNT bundles that recovers upon unloading. The experimental technique uses dynamic force transducers to measure stresses and a highsensitivity geometric moiré fringes system as non-contact dynamic displacement transducer. A microscopic high-speed camera is used for visual observation and in-situ characterization of the fundamental dynamic deformation mechanisms. This instrument can be used as a specialized method to study the rate-sensitive dynamic behavior of soft materials with complex micro-scale architectures. With fewer modifications to the loading apparatus, dynamic indentation experiments with time-resolved indentation depth information can be performed for rate-sensitive metals and other stiff/rigid hierarchical materials.

Acknowledgments We thank Prof. G. Ravichandran (California Institute of Technology) and Prof. M. Mello (Georgia Institute of Technology) for their useful suggestions. We acknowledge financial support from the Institute for Collaborative Biotechnologies (ICB) under the contract W911NF-09-D-0001 with the Army Research Office (ARO).

\section{References}

1. Lakes R (1993) Materials with structural hierarchy. Nature 36:511-515

2. Mayer G (2005) Rigid biological systems as models for synthetic composites. Science 310:1144-7. doi:10.1126/science.1116994

3. Ortiz C, Boyce M (2008) Bioinspired structural materials. Science 319:1053-1054. doi:10.1126/science. 1154295

4. Lee J-H, Singer JP, Thomas EL (2012) Micro-/nanostructured mechanical metamaterials. Adv Mater 24:4782-810. doi:10.1002/adma. 201201644

5. Maldovan M, Ullal CK, Jang J-H, Thomas EL (2007) Submicrometer scale periodic porous cellular structures: Microframes Prepared by holographic interference lithography. Adv Mater 19: 3809-3813. doi:10.1002/adma.200700811

6. Yuan Z-Y, Su B-L (2006) Insights into hierarchically mesomacroporous structured materials. J Mater Chem 16:663. doi:10. 1039/b512304f

7. Shim J, Shan S, Košmrlj A et al (2013) Harnessing instabilities for design of soft reconfigurable auxetic/chiral materials. Soft Matter 9: 8198. doi:10.1039/c3sm51148k
8. Purslow PP, Wess TJ, Hukins DWL (1997) Collagen Orientation and molecular spacing during creep and stress-relaxation in soft connective tissues. J Exp Biol 201:135-142

9. Lattanzi L, Raney JR, De Nardo L et al (2012) Nonlinear viscoelasticity of freestanding and polymer-anchored vertically aligned carbon nanotube foams. J Appl Phys 111:074314. doi:10.1063/1. 3699184

10. Yao H-B, Fang H-Y, Wang X-H, Yu S-H (2011) Hierarchical assembly of micro-/nano-building blocks: Bio-inspired rigid structural functional materials. Chem Soc Rev 40:3764-85. doi:10.1039/ $\mathrm{c} 0 \mathrm{cs} 00121 \mathrm{j}$

11. Teo EHT, Yung WKP, Chua DHC, Tay BK (2007) A carbon nanomattress: A new nanosystem with intrinsic, tunable, damping properties. Adv Mater 19:2941-2945. doi:10.1002/adma.200700351

12. Daraio C, Nesterenko VF, Jin S et al (2006) Impact response by a foamlike forest of coiled carbon nanotubes. J Appl Phys 100:064309. doi:10.1063/1.2345609

13. Lee J-H, Veysset D, Singer JP et al (2012) High strain rate deformation of layered nanocomposites. Nat Commun 3:1164. doi:10.1038/ ncomms 2166

14. Field JE, Walley SM, Proud WG, Goldrein HT, Siviour CR (2004) Review of experimental techniques for high rate deformation and shock studies. Int J Impact Eng 30:725-775. doi:10.1016/j.ijimpeng. 2004.03.005

15. Ramesh KT (2008) Springer handbook of experimental solid mechanics: High rates and impact experiments. Springer, New York

16. Espinosa HD, Nemat-Nasser S (2000) ASM Handbook: Lowvelocity impact testing. 539-559, American Society for Metals

17. Sutton MA, Orteu JJ, Schreier HW (2009) Image correlation for shape, motion and deformation measurements: Basic concepts, theory and applications. Springer, New York

18. Pan B, Qian K, Xie H, Asundi A (2009) Two-dimensional digital image correlation for in-plane displacement and strain measurement: A review. Meas Sci Technol 20(062001):17pp. doi:10.1088/09570233/20/6/062001

19. Hild F, Roux S (2006) Digital image correlation: From displacement measurement to identification of elastic properties - a review. Strain 42(2):69-80. doi:10.1111/j.1475-1305.2006.00258.x

20. Guduru PR, Rosakis AJ, Ravichandran G (2001) Dynamic shear bands: An investigation using high speed optical and infrared diagnostics. J Mech Mater 33:371-402. doi:10.1016/S0167-6636(01) 00051-5

21. Kajberg J, Sundin KG, Melin LG, Ståhle P (2004) High strain-rate tensile testing and viscoplastic parameter identification using microscopic high-speed photography. Int J Plast 20:561-575. doi:10.1016/ S0749-6419(03)00041-X

22. Koerber H, Xavier J, Camanho PP (2010) High strain rate characterisation of unidirectional carbon-epoxy IM7-8552 in transverse compression and in-plane shear using digital image correlation. J Mech Mater 42(11):1004-1019. doi:10.1016/j.mechmat.2010.09.003

23. Hutchens SB, Hall LJ, Greer JR (2010) In situ mechanical testing reveals periodic buckle nucleation and propagation in carbon nanotube bundles. Adv Funct Mater 20:2338-2346. doi:10.1002/adfm. 201000305

24. Professional Plastics, http://www.professionalplastics.com/ professionalplastics/content/Acetal_Delrin.pdf

25. Lu J, Suresh S, Ravichandran G (2003) Dynamic indentation for determining the strain rate sensitivity of metals. J Mech Phys Solids 51:1923-1938. doi:10.1016/j.jmps.2003.09.007

26. Oliver WC, Pharr GM (1992) An improved technique for determining hardness and elastic modulus using load and displacement sensing indentation experiments. J Mater Res 7(6):1564-1583. doi:10. 1557/JMR.1992.1564

27. Qi HJ, Teo KBK, Lau KKS, Boyce MC, Milne WI, Robertson J, Gleason KK (2003) Determination of mechanical properties of carbon nanotubes and vertically aligned carbon nanotube forests using 
nanoindentation. J Mech Phys Solids 51:2213-2237. doi:10.1016/j. jmps.2003.09.015

28. He LH, Fujisawa N, Swain MV (2006) Elastic modulus and stressstrain response of human enamel by nano-indentation. Biomaterials 27:4388-98. doi:10.1016/j.biomaterials.2006.03.045

29. Hay J (2009) Introduction to instrumented indentation testing. Exp Tech 33:66-72. doi:10.1111/j.1747-1567.2009.00541.x

30. Subhash G, Koeppel BJ, Chandra A (1999) Dynamic indentation hardness and rate sensitivity in metals. J Eng Mater Technol 121(3): 257-263. doi:10.1115/1.2812373

31. Misra A, Raney JR, De Nardo L, Craig AE, Daraio C (2011) Synthesis and characterization of carbon nanotube - polymer multilayer structures. 5(10):7713-21. doi: 10.1021/nn202262j

32. PCB Piezotronics, http://www.pcb.com/Products.aspx?m=200B02\#. UmXHW2B5-kI

33. PCB Piezotronics, http://www.pcb.com/Products.aspx?m=200B03\#. UmXHDGB5-kI

34. Davies EDH, Hunter SC (1963) The dynamic compression testing of solids by the method of the split Hopkinson pressure bar (SHPB). J Mech Phys Solids 11(3):155-179. doi:10.1016/0022-5096(63)90050-4

35. Song B, Chen W (2004) Dynamic stress equilibration in split hopkinson pressure bar tests on soft materials. Exp Mech 44:300-312. doi: $10.1177 / 0014485104041543$
36. Post D, Han B, Ifju P (1994) High sensitivity moiré: Experimental analysis for mechanics and materials. Springer, New York

37. Patorski K (1993) Handbook of the moiré fringe technique. Elsvier, New York

38. Cloud G (1998) Optical methods for engineering analysis. Cambridge University Press, Cambridge

39. Walker CA (1994) A historical review of moiré interferometry. Exp Mech 34(4):281-299. doi:10.1007/BF02325143

40. Raney JR, Misra A, Daraio C (2011) Tailoring the microstructure and mechanical properties of arrays of aligned multiwall carbon nanotubes by utilizing different hydrogen concentrations during synthesis. Carbon 49:3631-3638. doi:10.1016/j.carbon.2011.04. 066

41. Cao A, Dickrell PL, Sawyer WG, Ghasemi-Nejhad MN, Ajayan PM (2005) Super-compressible foamlike carbon nanotube films. Science 310:1307-1310. doi:10.1126/science.1118957

42. Suhr J, Victor P, Ci L et al (2007) Fatigue resistance of aligned carbon nanotube arrays under cyclic compression. Nat Nanotechnol 2:41721. doi:10.1038/nnano.2007.186

43. Misra A, Raney JR, De Nardo L, Craig AE, Daraio C (2011) Synthesis and characterization of carbon nanotube -polymer multilayer structures. ACS Nano 5:7713-21. doi:10.1021/ $\mathrm{nn} 202262 \mathrm{j}$ 